\title{
AVALIAÇÃO DO IMPACTO DAS INFECÇÕES HOSPITALARES (IHS) NA SAÚDE PÚBLICA E OS DESAFIOS DE CONTROLE E PREVENÇÃO
}

DOI: 10.48140/digitaleditora.2021.005.10

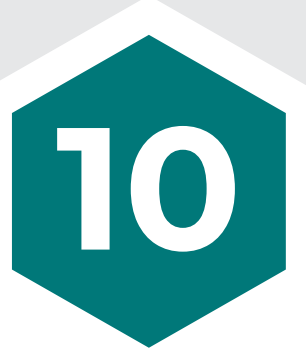

RESUMO

Objetivos: Desenvolver uma análise crítica do impacto das Infecções Hospitalares na Saúde Pública e seus desafios para controle e prevenção.

Métodos: Trata-se de um estudo de reflexão acerca dos desafios para controle e prevenção das infecções Hospitalares na Saúde Pública.

Resultados: Estima-se que no Brasil cerca de 5\% a 15\% dos pacientes internados contraem algum tipo de infecção hospitalar. essa infecção hospitalar pode crescer e agravar em média 5 a 10 dias ao período de internação.

Conclusão: É gigantesco o risco de contaminação por infecções hospitalares e seu impacto na Saúde Pública. É de suma importância um estudo aprofundando sobre o acometimento destas infecções, por trazer grandes problemas na saúde pública, afetando os profissionais de saúde e os pacientes.

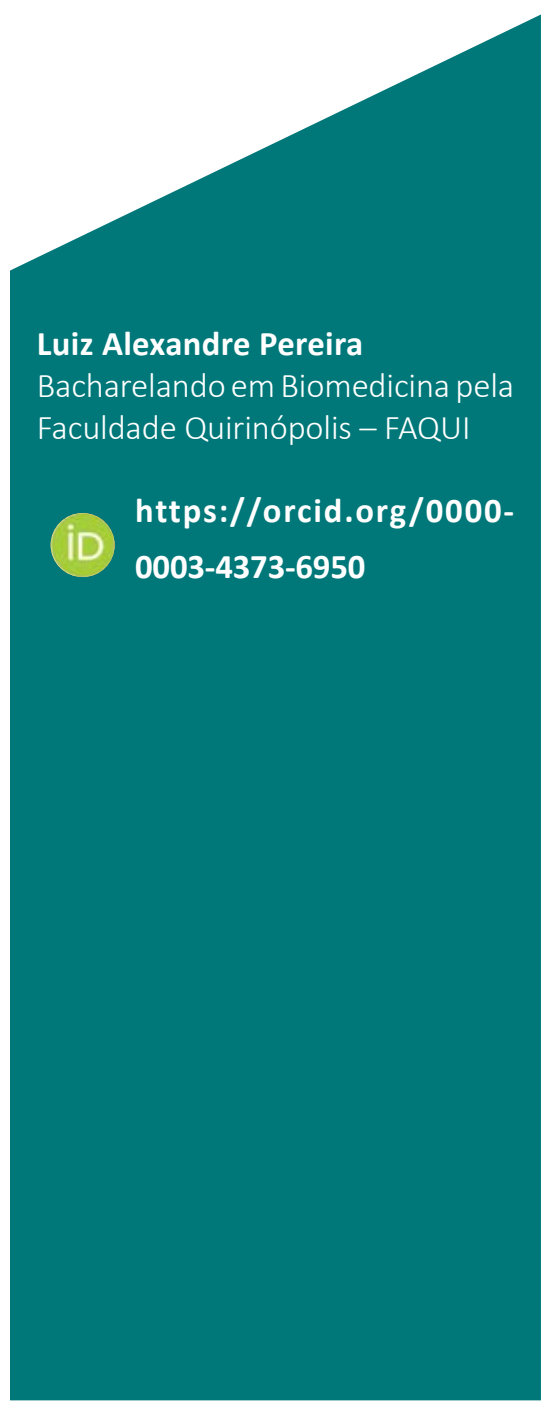

Palavras-chave Prevenção. Profissionais da Saúde. Assistência. Saúde Pública. 


\section{ASSESSMENT OF THE IMPACT OF HOSPITAL INFECTIONS (IHS) ON PUBLIC HEALTH AND THE CHALLENGES OF CONTROL AND PREVENTION}

DOI: $10.48140 /$ digitaleditora.2021.005.10

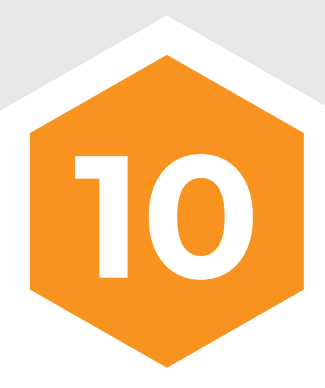

\section{ABSTRACT}

Recebido em: 30/11/2020

Aprovado em: 10/12/2020

Conflito de Interesse: não

Suporte Financeiro: não houve
Objectives: To develop a critical analysis of the impact of Hospital Infections on Public Health and its challenges for control and prevention.

Methodology: This is a study of reflection on the challenges for the control and prevention of nosocomial infections in public health.

Results: It is estimated that in Brazil about $5 \%$ to $15 \%$ of hospitalized patients contract some type of hospital infection. this nosocomial infection can grow and aggravate an average of 5 to 10 days to the hospitalization period.

Conclusion: The risk of contamination by hospital infections and its impact on Public Health is enormous. It is of utmost importance to study in depth the involvement of these infections, as it brings major problems in public health, affecting health professionals and patients. 


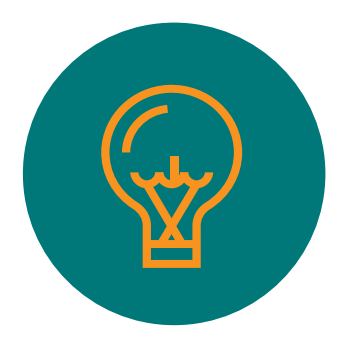

\section{INTRODUÇÃO}

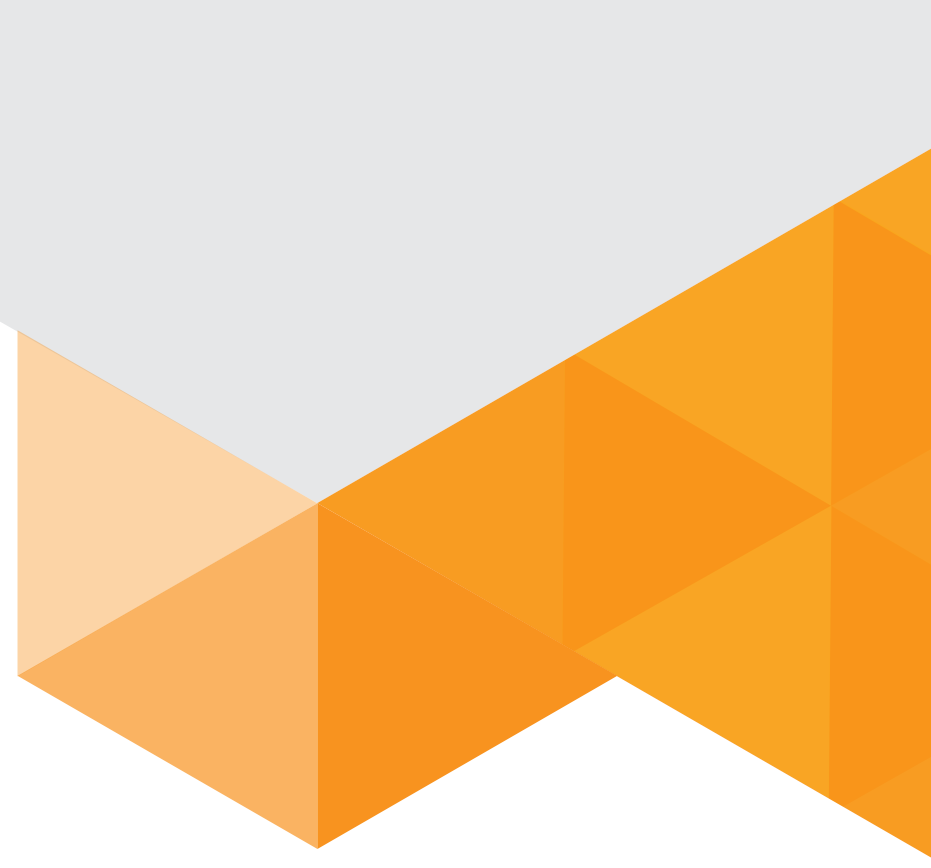

A infecção hospitalar (IH) é reconhecida mundialmente como um importante problema de saúde pública, devido a sua relação com o aumento da mortalidade hospitalar e geral, incapacidade física temporária ou permanente, o que ocasiona grandes custos diretos e indiretos (DA CRUZ PADRÃO et al., 2010).

É também considerada como uma iatrogenia relacionada com o grau de desenvolvimento dos hospitais, equipamentos, uso indiscriminado dos antimicrobianos, causando impacto humano, econômico e social (DA CRUZ PADRÃO et al., 2010).

IH é considerada como toda infecção que se desenvolve após a internação ou é produzida por microrganismos adquiridos durante a hospitalização do paciente, sendo excluídas as infecções que já estavam presentes ou no período de incubação à admissão (GUSMÃO, 2008).

Estas infecções podem ser de origem endógena, quando são causadas pela microbiota do paciente, ou exógena "quando resultam da transmissão a partir de fontes externas ao paciente (GUSMÃO, 2008).

Nesse sentido, as infecções relacionadas com a assistência em saúde, em unidades hospitalares, ambulatoriais ou até mesmo domiciliares, constituem um grave problema para os serviços responsáveis por essas modalidades de assistência e um desafio constante para a garantia da qualidade e segurança da atenção à saúde (CUNHA; COHEN, 2016).

Por esses motivos, torna-se imprescindível o estudo aprofundado do acometimento das infecções hospitalares na saúde com embasamento nas suas devidas e desafiantes prevenções. O objetivo principal deste estudo é revisar acometimento das infecções hospitalares e avaliar o impacto das infecções hospitalares na Saúde Pública. Especificamente o estudo teve como objetivos: compreender os desafios da prevenção das infecções hospitalares, identificar as principais formas de prevenção e demostrar a prevalência das infecções hospitalares. 


\section{DESENVOLVIMENTO}

Na Idade Média iniciaram-se as suspeitas de que algo "sólido" pudesse transmitir doenças de um indivíduo a outro. Foi em 1546 que Francastorius, defendeu a teoria de que certas patologias se transmitiam através de corpúsculos que ele denominou de semente da moléstia (seminária prima) e essas sementes transitavam de um corpo para outro através do contato direto ou através de roupas e objetos (COSTA, 2017).

De acordo com Gaspar, Busato e Severo (2012) a expressão Infecção Hospitalar (IH) só tenha sido utilizada na literatura médica brasileira a partir da década de 1970, desde 1950, textos científicos já demonstravam que a transmissão de doenças infecciosas no hospital era uma preocupação dos profissionais de saúde.

Em 1863, a enfermeira Florence Nightingale descreveu procedimentos de cuidados relacionados aos pacientes e ao ambiente, com a finalidade de diminuir os riscos da Infecção Hospitalar (IH). Florence solicitava que as enfermeiras mantivessem um sistema de relato dos óbitos hospitalares com o objetivo de avaliar o serviço (COSTA, 2017).

Temas como infecções no pós-operatório, técnicas assépticas, desinfetantes, esterilizantes, ar condicionado na sala cirúrgica, o problema do lixo nos hospitais e até mesmo a resistência microbiana já permeavam publicações médicas e de enfermagem na metade do século passado (GASPAR; BUSATO; SEVERO, 2012).

De acordo com Padoveze e Fortaleza (2014) os danos infecciosos da assistência foram trazidos à tona durante a chamada "revolução pasteuriana", por nomes como Ignaz Semmelweis, Florence Nightingale e Joseph Lister. Ao longo do século XX, em consequência do suporte avançado de vida e de terapias imunossupressoras, observou-se a necessidade de medidas de controle nos hospitais. Assim, as infecções hospitalares passaram a ser combatidas de forma sistemática nos países desenvolvidos.

O Ministério da Saúde define infecção hospitalar como aquela adquirida após a admissão do paciente e cuja manifestação ocorreu durante a internação ou após a alta, podendo ser relacionada com a internação ou procedimentos hospitalares (VILLAS BÔAS; RUIZ, 2004).

Desde meados da década de 1990, o termo "infecções hospitalares" foi substituído por "infecções relacionadas à assistência em saúde" (IRAS), sendo essa designação uma ampliação conceitual que incorpora infecções adquiridas e relacionadas à assistência em qualquer ambiente (PADOVEZE; FORTALEZA, 2014).

Analisando de forma geral, a infecção pode ser definida como uma invasão por micro-organismos nocivos, que vão além da capacidade de reação do organismo afetado e após infecção irão se multiplicar afetando os órgãos de acordo com a sua espécie e virulência (CABRAL; SILVA, 2013). 
Figura 01. Ciclo de Infecção

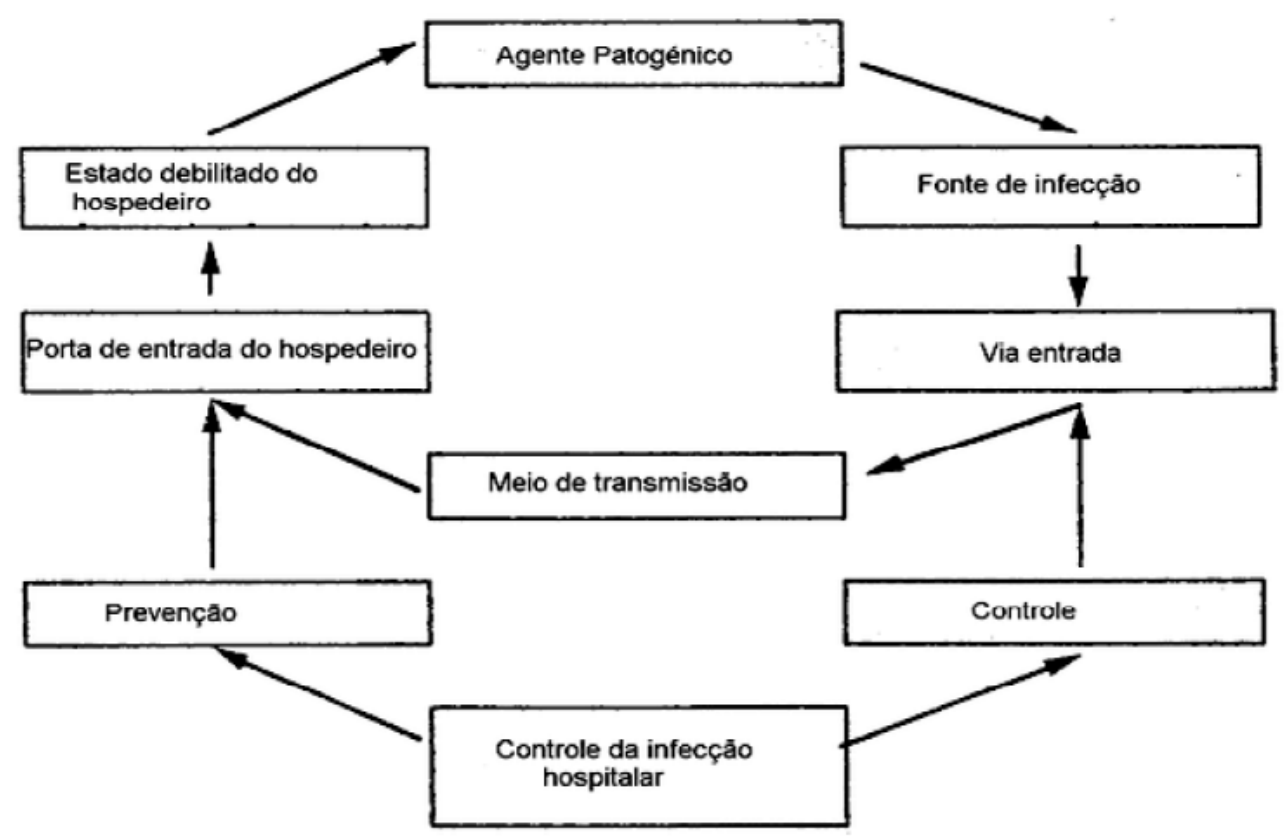

Fonte: adaptado de Cabral e Silva (2013)

Paiva et al. (2015) definem IH, como doenças que envolvem microrganismos (bactérias, fungos, vírus, etc.), inicialmente ocorre à penetração do agente infeccioso no corpo do doente e há multiplicação dos microrganismos, com consequente apresentação de sinais e sintomas. As infecções podem acometer diversas áreas do corpo, ou disseminar na corrente sanguínea.

Estima-se que entre 5 a 10\% dos pacientes internados possam ser diagnosticados com algum tipo de infecção durante o período de internação, pois existem diversos tipos de infecções (CABRAL; SILVA, 2013).

Tabela 1: Infecções hospitalares mais frequentes

\begin{tabular}{|c|c|}
\hline \multicolumn{2}{|r|}{ INFECÇÕES HOSPITALARES MAIS FREQUENTES } \\
\hline Infecções respiratórias & $\begin{array}{l}\text { Ocorrem em cerca de } 19 \% \text { dos casos. É certo que as porcetantagens de doentes } \\
\text { com esta patologia é bem superior, mas já são internados com ela. As suas cau- } \\
\text { sas são a flora nosocomial e a flora patogênica do doente. A principal incidência } \\
\text { é nos doentes com faixa etária compreendida entre } 53 \text { e } 64 \text { anos de idade. São } \\
\text { agravados pelo estado físico, mobilidade do doente, idade avançada. Muitos } \\
\text { destes casos resultam em morte. }\end{array}$ \\
\hline $\begin{array}{l}\text { Infecção por cateter } \\
\text { (flebite) }\end{array}$ & $\begin{array}{l}\text { Representa 13\% dos casos. Ocorrem ao manuseio necessário dos acessos veno- } \\
\text { sos. Consideram atos ivasivos todos os procedimentos que rompem a barreira } \\
\text { natural de proteção (pele), no entanto podem ser minimizados com um correto } \\
\text { procedimento. Podem aparecer devido à flebite, infecção relacionada e obstru- } \\
\text { ção do cateter. }\end{array}$ \\
\hline Infecção urinária & $\begin{array}{l}\text { Representam 34\% dos casos. Também devido à flora nosocomial e à flora do do- } \\
\text { ente, mais particularmente à flora intestinal. A propagação de microorganismos } \\
\text { deve-se em grande parte a uma técnica de assepsia, utilização indiscriminado } \\
\text { e abusivo do cateterismo, trauma durante e após o processo entre outros. A uti- } \\
\text { lização de gel urológico permite um melhor cateterismo auxilia na prevenção } \\
\text { destes traumas diminuindo o risco de infeção. }\end{array}$ \\
\hline Infecção da satura & $\begin{array}{l}\text { Aparecem em 17\% dos casos. Mais uma vez devem-se à flora patogênica do } \\
\text { doente e à flora nosocomial. No entanto, podem ocorrer devido a ultilização de } \\
\text { produtos químicos para assepsia da pele e má técnica de sutura e realização do } \\
\text { curativo. Podem ser agravadas por pela existência anterior de cirurgias e pelos } \\
\text { fatores anteriormente descrito. }\end{array}$ \\
\hline
\end{tabular}

Fonte: Adaptado de Cabral e Silva (2013) 
Diante desses problemas, percebe-se a importância da intervenção, já que a maioria das causas infecciosas é originada durante o cuidado direto com o paciente. Por esse motivo, todos os profissionais de saúde devem promover um ambiente seguro para todos (CABRAL; SILVA, 2013).

Embora as primeiras comissões de controle de infecção hospitalar (CCIH) tenham surgido na década de 1960, as ações governamentais programáticas no País tiveram início nos últimos anos da ditadura militar por meio de normativas do Ministério da Saúde (MS) (PADOVEZE; FORTALEZA, 2014).

Tal serviço deve ser composto por representantes das áreas de enfermagem, medicina, farmácia, microbiologia e administração, visando controlar as complicações infecciosas por meio de vigilância epidemiológica, a fim de investigar surtos de infecções, implementar intervenções, bem como avaliar a eficácia das mesmas (COSTA, 2017).

O Serviço de Controle de Infecção Hospitalar do referido hospital atua juntamente com uma equipe interdisciplinar, sendo responsável pela: a) vigilância epidemiológica das infecções, que conforme a Portaria 2.616/98-MS realiza observação ativa, sistemática e contínua da ocorrência e da distribuição dessas infecções entre os pacientes hospitalizados, bem como dos eventos e condições que afetam o risco de sua ocorrência. Acompanha diariamente a evolução dos pacientes internados, por meio de uma ficha de controle de $\mathrm{IH}$, registrando os procedimentos e fatores de risco do paciente internado; b) utilização do Antibiograma Escalonado, em que o laboratório libera o resultado ao corpo clínico apenas dos antibióticos do primeiro grupo aos quais as bactérias demonstraram sensibilidade. O resultado completo do antibiograma é encaminhado à CCIH que pode disponibilizá-lo ao interessado. Tal procedimento facilita a aderência do corpo clínico para controlar a utilização de antibióticos na instituição; c) capacitação dos profissionais de saúde quanto ao controle e prevenção de $\mathrm{IH}$. d) monitoramento das prescrições de antibióticos por meio de cópias dos antibiogramas que são disponibilizados à CCIH (GASPAR; BUSATO; SEVERO, 2012).

A prevenção da Infecção Hospitalar depende muito mais da instituição e de seus funcionários, do que dos pacientes e é conseguida através de medidas relativamente simples, porém essenciais, de realização imprescindível na rotina de qualquer hospital (CUNHA; COHEN, 2016).

Higienização das mãos é reconhecida mundialmente como medida primária muito importante no controle de infecções relacionadas à assistência à saúde, sendo considerada, um dos fatores essenciais na prevenção, controle de infecções dentro dos serviços de saúde e reduzindo a morbimortalidade (DE MELO; DE MOURA LEAL, 2015).

Os acidentes ocupacionais que ocorrem em hospitais estão relacionados a diversos fatores e, portanto, seu controle depende de ações em várias áreas, priorizando-se o desenvolvimento de divulgação de informações, além da adoção de procedimentos correspondentes às boas práticas de segurança para profissionais, pacientes e meio ambiente. As barreiras utilizadas na prevenção de riscos são Equipamentos de Proteção Individual (EPI), Equipamentos de Proteção Coletiva (EPC), adoção de medidas preventivas e imunização (CABRAL; SILVA, 2013).

A prevenção e o controle dessas infecções dependem, dentre outras medidas, da adesão e da motivação do profissional de saúde (MOTA, et al., 2014). 
AVALIAÇÃO DO IMPACTO DAS INFECÇÕES HOSPITALARES (IHS) NA SAÚDE PÚBLICA E OS DESAFIOS DE CONTROLE E PREVENÇÃO

No Figura 03, Cunha e Cohen (2016) apresenta dez práticas que podem diminuir os riscos de infecção hospitalar.

Figura 03: Dez práticas que podem diminuir os riscos de infecção hospitalar (CUNHA; COHEN, 2016).

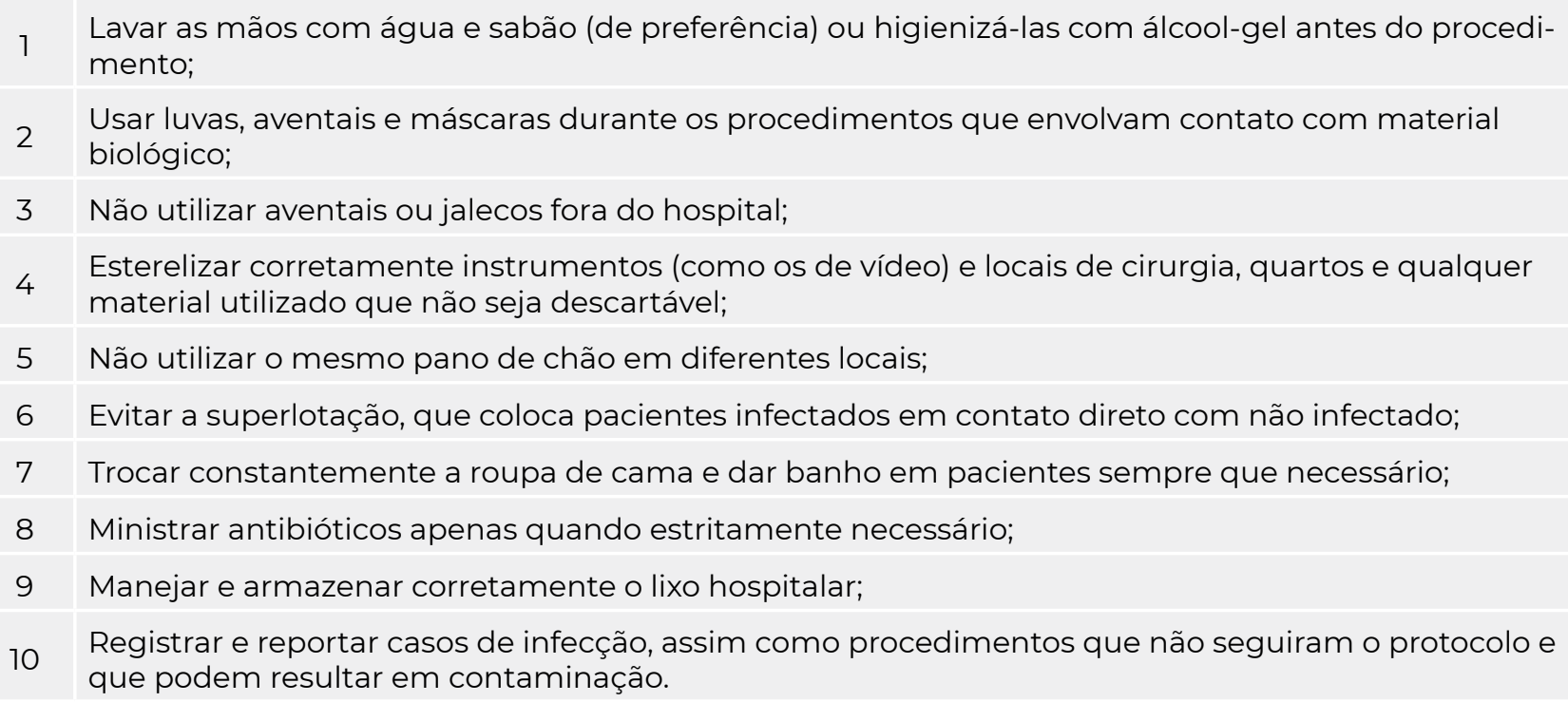

Fonte: Adaptado de Cunha e Cohen (2016)

No Brasil, os dados sobre Infecção Hospitalar são pouco divulgados. Além disso, esses dados não são consolidados por muitos hospitais, o que dificulta o conhecimento da real dimensão do problema (GASPAR; BUSATO; SEVERO, 2012).

Estima-se que no Brasil cerca de $5 \%$ a $15 \%$ dos pacientes internados contraem algum tipo de infecção hospitalar. Essa infecção hospitalar pode crescer e agravar em média 5 a 10 dias ao período de internação (SANTOS, 2016).

A incidência de infecções relacionadas à assistência à saúde associada a micro-organismos resistentes tem aumentado em todo o mundo. As Infeç̧ões nosocomiais têm sido um sério problema de saúde pública que afeta de 3\% a 6\% dos pacientes hospitalizados nos Estados Unidos, com isso resulta no maior período de internação, custos hospitalares e uma estimativa de 20.000 óbitos por ano. Nos Estados Unidos, representa a sexta causa de óbitos por ano. No Brasil, a taxa é de 1,5\% a 15\%, o que resulta em cerca de 45.000 óbitos e prejuízo da ordem de bilhões de reais anualmente (DE MELO; DE MOURA LEAL, 2015).

Houve um estudo realizado no país, ainda em 1994, avaliando 8.624 pacientes com mais de $24 \mathrm{~h}$ de internação e tempo médio de permanência de 11,8 dias. Foram identificados 1.129 pacientes com IH, representando uma taxa de 15,5\% (GASPAR; BUSATO; SEVERO, 2012).

A OMS, com base em dados de pesquisas, considera que 1,4 milhões de infecções ocorrem a qualquer momento, tanto nos países desenvolvidos quanto em países em desenvolvimento no Brasil e no mundo é avaliada como um problema crítico, gerando problemas sociais e econômicos (COSTA, 2017). 


\section{CONCLUSÃO}

Com base neste estudo, podemos notar o quão é gigantesco o risco de contaminação por infecções hospitalares. E é de suma importância o estudo aprofundando sobre o acometimento destas infecções, por trazer grandes problemas na saúde pública, afetando os profissionais de saúde e os pacientes.

A principal arma e saída para combater as IH ou IRAS é a prevenção, onde o treinamento dos profissionais de saúde é basicamente primordial para amenizar e banir este grande problema. As medidas simples como lavagem das mãos de forma correta, uso de EPI's, o cuidado para desinfecção dos equipamentos e ambientes, pode mudar a realidade atual. Entretanto, vimos que há um crescimento nas $\mathrm{IH}$, devido à falta de adesão dessas medidas, seja por descaso, falta de investimento ou ausência de implantação das $\mathrm{CClH}$. 


\section{REFERÊNCIAS}

CABRAL, Francisco Willians; SILVA, Maria Zildenia Oliveira. Prevenção e controle de infecções no ambiente hospitalar. SANARE-Revista de Políticas Públicas, v. 12, n. 1, 2013.

COSTA, Matheus Fernandes Santos. Infecção Hospitalar em pessoas idosas em um hospital universitário do Distrito Federal. Trabalho de Conclusão de Curso, Universidade de Brasília, Faculdade de Ciências da Saúde, Brasília, 2017.

CUNHA, Esdras Barros; COHEN, Juliana Vieira Frezza Bernardes. Aspectos relevantes da prevenção e controle de infecções hospitalares. Revista Saber Cientifico, v. 1, n. 1, 2016.

DA CRUZ PADRÃO, Manuella et al. Prevalência de infecções hospitalares em unidade de terapia intensiva. Revista da Sociedade Brasileira de Clínica Médica, v. 8, n. 2, p. 125-8, 2010.

DE MELO, Mickaely Horrara Craveiro; DE MOURA LEAL, Adinaide Cristina Almondes. Controle das infecções na assistência à saúde relacionada à higienização das mãos. Revista Interdisciplinar, v. 8, n. 1, p. 91-97, 2015.

GASPAR, Maria Dagmar; BUSATO, Cesar Roberto; SEVERO, Emanoel. Prevalência de infecções hospitalares em um hospital geral de alta complexidade no município de Ponta Grossa. Acta Scientiarum. Health Sciences, v. 34, n. 1, p. 23-29, 2012.

GUSMÃO, Maria Enoy Neves. Infecção Hospitalar: mortalidade, sobrevida e fatores prognósticos no Hospital Universitário Professor Edgard Santos/UFBA. Trabalho de Conclusão de Curso (pós-graduação), Universidade Federal da Bahia, Salvador, 2008.

MOTA, Ercília Campos et al. Higienização das mãos: uma avaliação da adesão e da prática dos profissionais de saúde no controle das infecções hospitalares. Rev Epidemiol Control Infect, v. 4, n. 1, p. 12-17, 2014.

PAIVA, Patrícia Alves et al. Medidas de prevenção e controle das infecções hospitalares em unidade de terapia intensiva. Revista da Universidade Vale do Rio Verde, v. 13, n. 2, p. 669-680, 2015.

SANTOS, Fernanda Scheylla Velame Dantas. Biossegurança em hospitais, prevenção e controle de Infecções: uma revisão. Trabalho de Conclusão de Curso, Faculdade Maria Milza, Governador Mangabeira, 2016.

VILLAS BÔAS, Paulo José Fortes; RUIZ, Tânia. Ocorrência de infecção hospitalar em idosos internados em hospital universitário. Revista de saúde pública, v. 38, p. 372-378, 2004. 